\title{
Cervical epidural anaesthesia for thyroid surgery
}

\author{
Khanna $\mathbf{R}^{1}$, Singh DK ${ }^{2}$
}

${ }^{1}$ Department of Surgery, ${ }^{2}$ Anaesthesiology, Institute of Medical Sciences, Banaras Hindu University, Varanasi, India.

\begin{abstract}
Background: Cervical epidural anaesthesia is a regional anaesthesia technique which has been used for upper limb surgery, upper thoracic wall surgery, carotid artery surgery and neck dissections. Anaesthesia for thyroid surgery can be complicated due to the altered functional status of the thyroid or its large size.

Objective: This prospective study was designed to assess the effectiveness and safety of cervical epidural anaesthesia for thyroid surgery.

Materials and methods: Cervical epidural anaesthesia was attempted in 9 patients and the results compared with 44 patients who underwent thyroid surgery under conventional general anaesthesia with endotracheal intubation. The epidural catheter was placed in the $\mathrm{C}_{7}-\mathrm{T}_{1}$ vertebral interspace and $10-15 \mathrm{ml}$ of $1 \%$ Lignocaine with adrenaline was injected.

Results: The technique of cervical epidural anaesthesia was successfully used in 8 out of 9 patients in whom it was attempted All patients were maintained in a state of conscious - sedation and effective analgesia was obtained in all 8 patients. There were no significant complications especially those related to diaphragmatic function and cardiovascular stability. In contrast patients undergoing surgery under conventional general anaesthesia had complications related to endotracheal intubation, cardiac arrhythmias and hypotension

Conclusion: The technique of cervical epidural anaesthesia should be considered in thyroid patients where difficult endotracheal intubation is anticipated and in those in whom alterations in thyroid functional state make them vulnerable to cardiovascular complications under conventional general anaesthesia.
\end{abstract}

Key words: thyroid surgery, cervical epidural anaesthesia

$\mathrm{P}$ oviding safe and effective anaesthesia for thyroid surgery can sometimes become a problem for the anaesthetists. This becomes especially relevant in large goitres which might compress or deviate the trachea. Endotracheal intubation is difficult in such cases and in large goitres can be hazardous. Also the goitre might be associated with thyroid functional disorders. Patients with both hypo and hyperthyroidism are prone to cardiac rhythm disorders which might get aggravated under the influence of general anaesthetic agents.

Regional anaesthetic techniques are safer than general anaesthesia(GA) in high risk patients where endotracheal intubation is difficult or cardiac arrhythmias are anticipated. For thyroid surgery regional anaesthesia is not a conventionally described option. Cervical epidural anaesthesia (CEA) has been used for shoulder, upper extremity, and thoracic wall surgery with good results. Here we describe our experiences of using this technique for thyroid surgery.

\section{Materials and methods}

The technique of cervical epidural anaesthesia was used in 8 patients who underwent thyroid surgery over an 24 months period from January 2003 to December 2004. Over the same period 44 patients underwent thyroid surgery under conventional general anaesthesia with endotracheal intubation. Details of both groups of patients are described in Table I.

Selection Criteria: The second author is a proponent of cervical epidural anaesthesia and used this technique in all cases who underwent thyroid surgery under his supervision. The other 44 operations were done under the supervision of other colleagues in the Department of Anaesthesiology who administered GA with endotracheal intubation.

Technique of CEA

The patient was placed in a sitting position with flexion at the neck and forehead supported on a table. Skin at the nape of neck was disinfected with cetrimide and

Correspondence

Dr. Rahul Khanna

A-15, Brij Enclave

Sunderpur, Varanasi - 221 005, India

E-mail: dr_rahul khanna@rediffmail.com 
spirit. The spine of the $\mathrm{C}_{7}$ vertebra was identified as the $1^{\text {st }}$ prominent spine while palpating in the midline from the occiput downwards. The space between the $\mathrm{C}_{7}$ and $\mathrm{T}_{1}$ vertebral spines was infiltrated with $2 \%$ Lignocaine. An 18 gauge Tuohy needle was inserted in the $\mathrm{C}_{7}-\mathrm{T}_{1}$ interspace perpendicularly so that the needle would be parallel to the vertebral spine. The bevel of the needle was kept facing upwards. The needle was advanced carefully to get a feel of piercing the ligamentum flavum . It should be kept in mind that the resistance offered by the ligament in the neck is lesser than in the lumbar area. Epidural placement of the needle tip was ascertained by loss of resistance or the hanging drop technique. An epidural catheter was guided through the Tuohy needle and advanced $3-4 \mathrm{~cm}$ in to the epidural space followed by removal of the needle. After reconfirming by aspiration that subarachnoid entry had not occurred $10-15 \mathrm{ml}$ of $1 \%$ Lignocaine with 1 : 200,000 Adrenaline was injected through the catheter. The patient was placed in supine position and after a 15 minutes observation of the pulse rate, respiratory effort and blood pressure, 'thyroid position' with extension of the neck was permitted.

\section{Results}

Out of the 9 patients in whom it was attempted CEA was successful in 8 . It was abandoned in one patient because of hemorrhagic aspirate through the Tuohy needle. This patient was operated under conventional general anaesthesia with endotracheal intubation. The other 8 patients were comfortably maintained sedated but awake during the procedure with no untoward effect. Monitoring of the pulse, respiratory effort, BP and peripheral oxygen saturation was carried out and all patients maintained these parameters without additional support. In 2 patients where the surgery lasted for more than 90 minutes, additional injection of $10 \mathrm{ml} 1 \%$ Lignocaine with adrenaline was required through the epidural catheter to maintain analgesic effect.

Out of the 44 patients who underwent thyroid surgery under GA, 6 had serious problems at the time of endotracheal intubation. In 3 , the endotracheal tube could not be passed and the patients were ventilated using 'laryngeal masks'. Other 3 patients were intubated after repeated efforts using narrow 6.5 Fr endotracheal tubes mounted on guide wires. These patients were kept intubated for 24 hours post operatively for the fear of laryngeal oedema caused by traumatic endotracheal intubation. Another 4 patients suffered arrhythmias intra-operatively which required cessation of $\mathrm{N}_{2} \mathrm{O}$ and correction with intravenous Lignocaine. Hypotension immediately after intubation was noticed in 11 patients including the 6 with difficult intubation.

Table 1: Profile of patients undergoing thyroid surgery under CEA \& GA

\begin{tabular}{|l|c|c|}
\hline & CEA $(\mathbf{n}=\mathbf{8})$ & GA (n= 44) \\
\hline Mean Age & 41 & 46 \\
\hline Sex Ratio & $(32-56)$ & $\mathrm{M}: \mathrm{F}:: 1: 4$ \\
\hline Thyroid Functional Status & $\mathrm{M}: \mathrm{F}:: 1: 3$ & 39 \\
Euthyroid & & 5 \\
Hyperthyroid & 7 & 09 \\
\hline Tracheal Compression / Deviation & 1 & 35 \\
Present & 2 & 30 \\
Absent & 6 & 14 \\
\hline Nature of surgery & 6 & 35 \\
Hemithyroidectomy & 2 & \\
Total thyroidectomy & & \\
\hline
\end{tabular}

Table 2: Comparison of results of CEA versus GA for thyroid surgery

\begin{tabular}{|l|c|c|}
\hline & CEA $\mathbf{( n = 9 )}$ & GA (n= 44) \\
\hline Successful technique & 08 & 38 \\
Technical difficulty & - & 03 \\
Conversion to another technique & 01 & 03 \\
Intra-operative arrhythmias & - & 04 \\
Intra-operative hypotension & - & 06 \\
Anaesthesia related mortality & Nil & Nil \\
\hline
\end{tabular}




\section{Discussion}

Providing safe anaesthesia for thyroid surgery can be a difficult task for two reasons (1) Possibility of tracheal deviation or compression because of enlarged thyroid, (2) Possibility of hemodynamic disturbances including arrhythmias and hypotension related to the functional status of the thyroid. Conventional general anaesthesia with endotracheal intubation may not always be possible and even hazardous in certain situations. Endotracheal intubation can become a traumatic procedure precipitating laryngeal oedema occasionally. Anaesthetic gases, Propofol and muscle relaxants can all become arrythmogenic in the presence of covert or even occult hypo or hyperthyroidism states. As in other situations where general anaesthesia becomes a high risk proposition, regional anaesthesia is considered the safer alternative. But for thyroid surgery, regional anaesthetic techniques have neither been adequately described nor practiced widely.

Cervical epidural anaesthesia aims to anesthetise the skin in front of the neck where the incision would be placed. The front of the neck is supplied by $2^{\text {nd }}$ and $3^{\text {rd }}$ cervical rami which form the transverse cutaneous nerve of the neck. The lower part of the neck and the part lateral to sternocleidomastoid muscle is supplied by supraclavicular nerves which arise from the $3^{\text {rd }}$ and $4^{\text {th }}$ cervical ventral rami. For delivering CEA, we introduced the epidural catheter in the $\mathrm{C}_{7}-\mathrm{T}_{1}$ vertebra interspace and guided it upwards by keeping the bevel of the Tuohy needle facing cranially. By using the technique described above, the upper level of sensory block obtained was at $\mathrm{C}_{2}$ level and the lower level varied from $T_{5}$ to $T_{10}$.

Cervical epidural anaesthesia has been used mainly for hand, upper limb, shoulder and upper thoracic wall surgery including mastectomy. It has also been used for carotid artery surgery ${ }^{1}$, parathyroid surgery ${ }^{2}$, neck dissection for head and neck cancers ${ }^{3}$ and for the treatment of complex regional pain syndromes of the upper $\operatorname{limb}^{4}$. Adequate surgical anaesthesia with a high degree of patient acceptance has been reported by all authors. Failure of the technique or the need to convert to an alternative method of anaesthesia has not been reported in the literature.

The main reasons why CEA has not come into wide clinical usage are fears of its potential complications. These include its effects on respiratory function especially phrenic nerve controlled diaphragmatic movements and cardio-vascular stability. Despite the concern over pulmonary dysfunction with CEA, it is rarely of clinical significance even in debilitated patients ${ }^{1}$. Phrenic nerve is the sole motor supply to the diaphragm and also contains wide spread sensory fibres.
It arises chiefly from the fourth cervical ramus but also contains contributions from the $3^{\text {rd }}$ and $5^{\text {th }}$. Being mainly a motor nerve it is exceptionally resistant to the effects of Lignocaine when it is used at a concentration of $1 \%$ and deployed in the epidural space. Stevens et al (5) found a measurable reduction in bedside pulmonary function test after CEA. They found that the forced expiratory volume (FEV) and forced vital capacity (FVC) decreased by $12-16 \%$ after using $300 \mathrm{mg}$ of Lignocaine but these changes were not clinically significant. They concluded that the motor block of phrenic nerve is incomplete after CEA. Capdevila et al ${ }^{6}$ using ultrasonography found that the diaphragmatic excursion decreased from 5.6 to 4.4 . $\mathrm{cm}$ after CEA. They found that although CEA provided painless rehabilitation, it affected diaphragmatic function and respiratory drive.

We found that CEA did not have any effect on respiratory functions. None of our patients had changes in breathing pattern or fall in peripheral arterial oxygen saturation on pulse oximetry. Acute paralysis of the diaphragm should produce violent contractions of the abdominal muscles as a compensatory mechanism. This was not found in any of our patients. Also none of our patients developed pulmonary atelectasis post operatively.

The other major concern with CEA has been its effects on heart rate and hemodynamic stability. CEA can result in cardiac sympathectomy which can depress phasic and tonic dynamic modulation of the cardiac cycle $^{(7)}$. This can lead to a lack of increase in heart rate and a decrease in mean arterial pressure. CEA has also been found to attenuate the pancuronium induced tachycardia as a result of cardiac sympathetic denervation ${ }^{8}$. The cardiovascular stimulatory effects of Ketamine are also suppressed partially by CEA ${ }^{9}$. The most frequently reported side effects of CEA are hypotension in $10.9 \%$ and bradycardia in $2.8 \%$

In our experience none of our patients including the one with hyperthyroidism demonstrated an adverse cardiovascular side effect. No patient required ionotropic support or an anti-arrhythmia medication. Among the patients operated under general anaesthesia, 6 had hypotension and 4 significant arrhythmias. These effects were specially marked in patients with difficult endotracheal intubation or hyperthyroid state. Thus the cardiac sympathetic denervation effect of CEA may be especially useful in patients with hyperthyroidism.

All our patients operated under CEA were maintained in a state of conscious - sedation. This allows continuous verbal communication with the patient and early detection of recurrent laryngeal nerve injury which can occur during thyroid surgery. 


\section{Conclusion}

Review of literature and our own experience shows that CEA is a safe and reliable technique. It can be easily learnt by anaesthetists who are already familiar with lumbar epidural anaesthesia. The complication rates are low and easily manageable. The risks of diaphragmatic paralysis and hemodynamic stability are present but rarely of clinical significance. We recommend this technique as the procedure of choice for thyroid surgery.

\section{References}

1. Bonnet F, Derosier JP, Pluskwa F, Abhay K, Gaillard A. Cervical epidural anaesthesia for carotid artery surgery. Canadian J Anaesthesiology. 1990;37(3):353 - 8.

2. Michalek P, David I, Adamec M, Janousek L. Cervical epidural anaesthesia for combined neck and upper extremity procedure: a pilot study. Anaesth Analg. 2004; 99(6):1833 - 6.

3. Wittich DJ Jr, Berny JJ, Davis RK. Cervical epidural anaesthesia for head and neck surgery. Laryngoscope. 1984. 94(5):615 - 9.

4. Buchheit T, Crews JC. Lateral cervical epidural catheter placement for continuous unilateral upper extremity analgesia and sympathetic block. Regional Anaesthesia and Pain Medicine. $2000 ; 25(3): 313-7$.
5. Stevens RA, Frey K, Sheikh T, Kao TC, Milcat - Stevens M, Morales M. Time course of the effects of cervical epidural anaesthesia on pulmonary function. Regional Anaesthesia \& Pain Medicine. 1998;23(1):20 -4.

6. Capdevila X, Biboulet $\mathrm{P}$, Rubenovitch J, Serre - Cousine O, Peray P, Deschodt J, et al. The effects of cervical epidural anaesthesia with bupivacaine on pulmonary function in conscious patients. Anaesthesia \& Analgesia. 1998;86(5):1033 - 8 .

7. Tanaka M, Goyagi T, Kimura T, Nishikana T. The effects of cervical and lumbar epidural anaesthesia on heart rate variability and spontaneous sequence baroreflex sensitivity. Anaesthesia \& Analgesia. 2004;99(3):924 - 9.

8. Omete K, Iwasaki H, Namiki A. Cervicothoracic epidural anaesthesia blunts pancuronium induced increase in heart rate in humans. Acta Anaesthesiologica Scandinavica. 1993;37(4):415 - 8 .

9. Mayumi T, Dohi S, Takahashi T. Cardiovascular effects of ketmine in humans with cervical or lumbar epidural blockade. Anaesthesiology. 1985;62(1): $39-43$. 